\title{
Traumatic Atlanto-Occipital Dislocation Presenting With Dysphagia as the Chief Complaint: A Case Report
}

\author{
Eun Hye Choi, $\mathrm{MD}^{1}$, Ah Young Jun, $\mathrm{MD}^{2}$, Eun Hi Choi, $\mathrm{MD}^{1}$, Ka Young Shin, $\mathrm{MD}^{1}$, Ah Ra Cho, MD \\ ${ }^{1}$ Department of Physical Medicine and Rehabilitation, Hallym University Chuncheon Sacred Heart Hospital, \\ Hallym University College of Medicine, Chuncheon; ${ }^{2}$ Department of Physical Medicine and Rehabilitation, \\ Hallym University Dongtan Sacred Heart Hospital, Hallym University College of Medicine, Hwaseong, Korea
}

We report a patient with traumatic atlanto-occipital dislocation who presented with dysphagia as the chief complaint. A 59-year-old man complained of swallowing difficulty for 2 months after trauma to the neck. On physical examination, there was atrophy of the right sternocleidomastoid and upper trapezius muscles, and the tongue was deviated to the right. In a videofluoroscopic swallowing study, penetration and aspiration were not seen, food residue remained in the right vallecula and pyriform sinus, and there was decreased motion of the soft palate, pharynx and larynx. Electromyography confirmed a right spinal accessory nerve lesion. Magnetic resonance imaging confirmed atlanto-occipital dislocation. Dysphagia in atlanto-occipital dislocation is induced by medullary compression and lower cranial nerve injury. Therefore, in survivors who are diagnosed with atlantooccipital dislocation, any neurological symptoms should be carefully evaluated.

Keywords Atlanto-occipital joint, Dysphagia, Cranial nerves

\section{INTRODUCTION}

Traumatic atlanto-occipital dislocation is an uncommon and usually fatal injury with an incidence of $0.7 \%-$ $1.3 \%$ of all cervical injuries [1]. It is usually caused by high energy trauma such as trauma a traffic accident or a

Received July 2, 2012; Accepted August 17, 2012

Corresponding author: Ah Young Jun

Department of Physical Medicine and Rehabilitation, Hallym University Dongtan Sacred Heart Hospital, Hallym University College of Medicine, 40 Seokwu-dong, Hwaseong 445-170, Korea

Tel: +82-31-8086-2350, Fax: +82-31-8086-2709, E-mail: ahyoungjun@ gmail.com

(c) This is an open-access article distributed under the terms of the Creative Commons Attribution Non-Commercial License (http://creativecommons. org/licenses/by-nc/3.0) which permits unrestricted noncommercial use, distribution, and reproduction in any medium, provided the original work is properly cited.

Copyright $\odot 2013$ by Korean Academy of Rehabilitation Medicine fall from a great height. The clinical presentation of those who survive atlanto-occipital dislocation varies. The patients who survive often have a neurologic deficit, but nearly $20 \%$ of patients will have a normal neurologic examination on presentation [2]. Atlanto-occipital dislocation is frequently missed at initial presentation because of its rarity [3].

Neurologic dysphagia may be caused by disruption of different parts of the central nervous system (e.g., the supranuclear level, the level of the motor and sensory nuclei taking part in the swallowing process, peripheral nerve level, or muscle cell and spindle cell pathology) or by neuromuscular and muscular disorders [4].

This report presents a case of traumatic atlanto-occipital dislocation presenting with dysphagia as the chief complaint. 

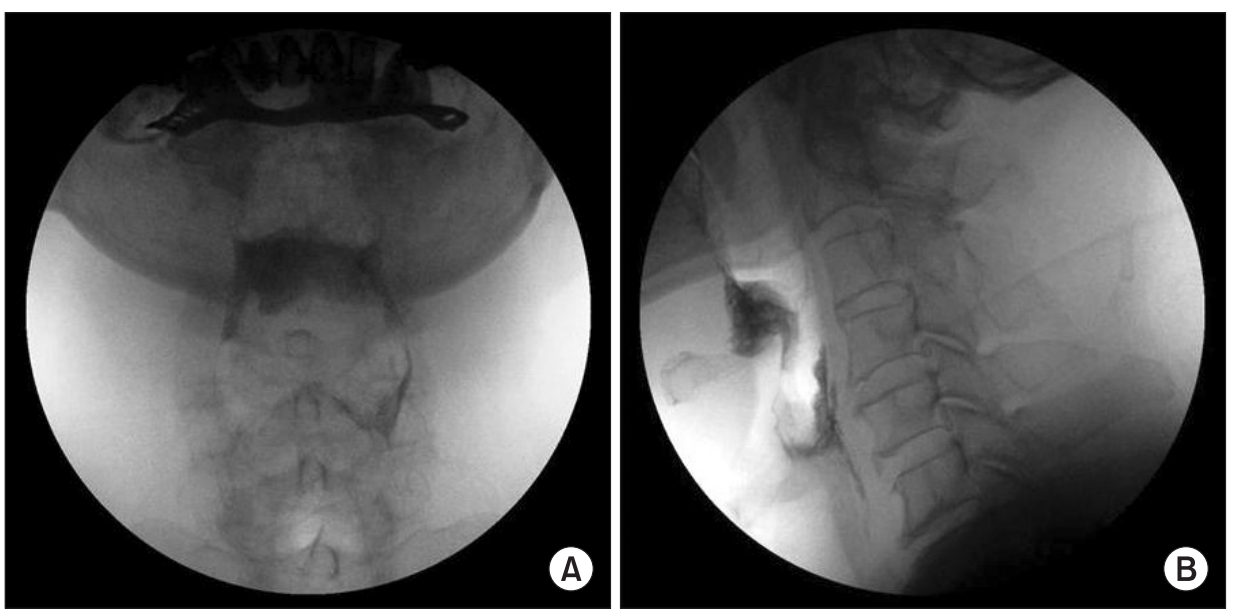

Fig. 1. (A) The anteroposterior projection of the videofluoroscopic image shows much barium residue in the right vallecula after swallowing the solid component. (B) The lateral projection shows much barium residue in the vallecula and pyriform sinus after swallowing the solid component.

\section{CASE REPORT}

A 59-year-old male visited the rehabilitation department because of ongoing difficulty swallowing. Two months earlier, a 15-kg piece of iron was dropped on his neck from a height of $10 \mathrm{~m}$. He was admitted and diagnosed with whiplash injury and a compression fracture of the fifth thoracic vertebra. He was treated conservatively, and the neck pain subsided. As the swallowing difficulty continued, he visited the rehabilitation department.

On physical examination, there was slight atrophy of the right sternocleidomastoid and upper trapezius muscles. The tongue was deviated to the right, and the gag reflex was impaired but the uvula was not significantly deviated. He complained of mild dysarthria and a hoarse voice. Examination of the upper and lower extremities revealed no weakness, but the power of right scapula elevation, head tilt to the right, and neck rotation to the left were decreased. The sensory examination was normal.

In a motor nerve conduction study, the compound muscle action potential of the right accessory nerve was not evoked. Needle electromyography revealed abnormal spontaneous activity in the right trapezius and sternocleidomastoid muscles. Injury of the accessory nerve was confirmed, although the other cranial nerves were not checked because the patient would not cooperate.

A videofluoroscopic swallowing study (VFSS) revealed that lip closure and jaw movement were normal, but the soft palate and tongue movements were impaired. There were decreased epiglottic inversion, laryngeal elevation and movement of pharyngeal wall and delayed pharyngeal transit time. There was no penetration or aspiration of liquid, semisolid, or solid, but a large amount of mate-

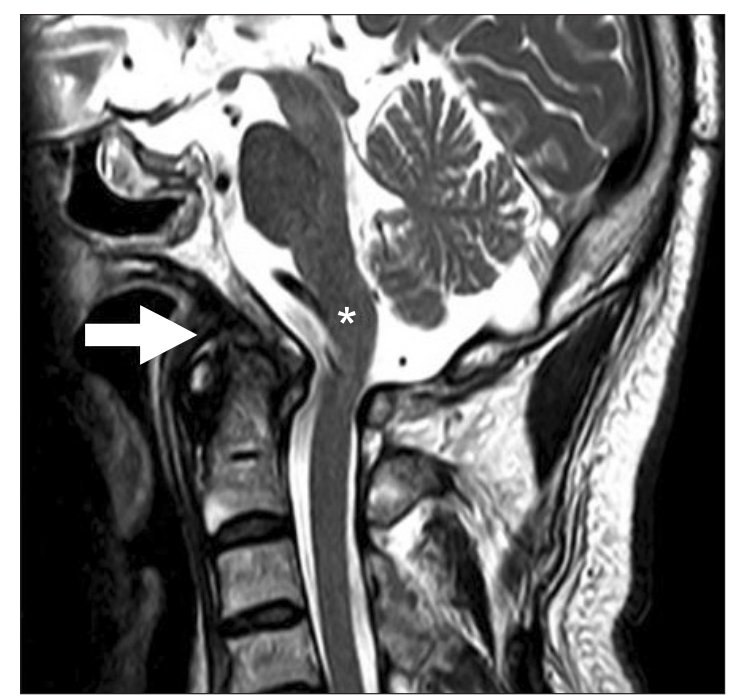

Fig. 2. The T2-weighted magnetic resonance imaging of the cervical spine in the sagittal section shows medullary compression (asterisk) and disruption of ligaments (arrow).

rial was retained in the right vallecula and pyriform sinus (Fig. 1).

To identify the cause of the dysphagia, brain and cervical magnetic resonance imaging (MRI) were performed. Brain MRI showed no abnormal findings in the brain parenchyma, and therefore, a brain lesion was excluded. Cervical MRI showed medullary compression and disruption of ligaments (Fig. 2). The sagittal section of the cervical computed tomography (CT) clearly showed a posteriorly displaced basion with respect to the tip of the dense of axis, and therefore, a posterior atlanto-occipital dislocation was diagnosed. And in the axial view, bony erosion of the atlas on the right side was shown (Fig. 

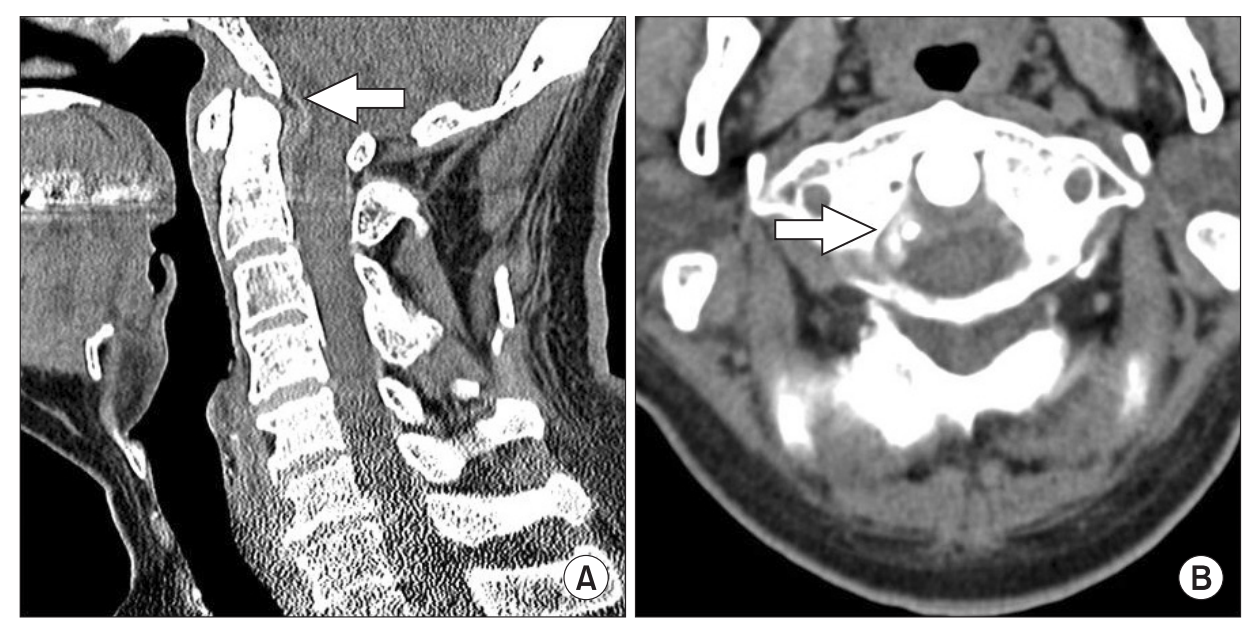

Fig. 3. On computed tomography, (A) the sagittal section shows the posteriorly displaced basion with respect to the tip of the dense of axis (arrow) and (B) the axial section shows bony erosion of the atlas on the right side (arrow).
$3)$. For the treatment of the posterior atlanto-occipital dislocation, surgery was recommended, but the patient refused. Consequently, he underwent occupational therapy for the dysphagia. We educated the patient on a compensatory strategy when swallowing (rotation of the neck to the right side while swallowing food) and advised him to have repeated swallows to reduce pharyngeal residues. And he was also encouraged to perform resistance and range of motion exercises for the oral tongue, tongue base, neck, and shoulders to improve swallowing function. We have followed him for one year, and his dysphagia symptoms (choking during liquid swallowing, sensation of retained residual after swallowing, and food leakage into the nasal cavity) have improved. But, the degree of atlanto-occipital dislocation which was evaluated by X-ray was not changed.

\section{DISCUSSION}

The atlanto-occipital joint has little intrinsic bony stability. The normal occipito-vertebral relationship is maintained by the ligaments. The anterior atlantooccipital, cruciate, apical and alar ligaments and tectorial membrane confer stability to the joint. Traynelis et al. [3] classified atlanto-occipital dislocations into three types: type 1 , anterior displacement of the occiput on the atlas; type 2, longitudinal distraction with separation of the occiput from the atlas; and type 3, posterior displacement of the occiput on the atlas, as in this case.

A variety of radiographic measurements have been purposed for the diagnosis of atlanto-occipital dislocation on a lateral cervical radiograph. Currently, a method proposed by Harris et al. [5] is the most reliable means to diagnosis atlanto-occipital dislocation on a lateral cervical radiograph. By their method, a displacement of more than $12 \mathrm{~mm}$ or more than $-4 \mathrm{~mm}$ between the basion and posterior C2 line, or a displacement of more than $12 \mathrm{~mm}$ from the basion to the dens is considered abnormal. In our case, the distance from the basion to posterior C2 line was $-4.5 \mathrm{~mm}$. Therefore we diagnosed posterior displacement of the occiput on the atlas.

There are numerous reports of patients developing atlanto-occipital dislocation due to trauma [1-3]. Traumatic atlanto-occipital dislocation is usually fatal, and survival without a neurological deficit is uncommon. Because the injuries are to the brain stem and the vascular structures of the neck in the case of traumatic atlanto-occipital dislocation, severe upper cervical injury is frequently associated with respiratory failure [6]. The diagnosis of atlantooccipital dislocation is suggested by several neurologic injuries after trauma. These neurologic deficits are lower cranial nerve paresis, monoparesis, hemiparesis, quadriparesis, respiratory dysfunction, and complete high cervical cord motor deficits [2]. In a patient with minor neurologic symptoms, the diagnosis of atlanto-occipital dislocation can be delayed or missed on initial radiology [3].

In our case, dysphagia was the chief complaint after atlanto-occipital dislocation. This symptom was due to the neurologic injury induced by atlanto-occipital dislocation. Survivors who are recovering from atlanto-occipital dislocation can also present with dysphagia due to retropharyngeal pseudomeningocele [7]. A pseudomeningocele develops as the result of extravasation of cerebral spinal fluid after a dural tear. It can present as respiratory distress or dysphagia and may develop weeks after the 
injury. For this reason, retropharyngeal pseudomeningocele should also be suspected in patients who survive atlanto-occipital dislocation [7].

Therefore, a meticulous clinical and imaging evaluation is essential to exclude atlanto-occipital problems in the patient with multiple injuries. CT and MRI are recommended for patients suspected of having atlanto-occipital dislocations to confirm the dislocation, identify any associated fractures, and assess the extent of ligamentous disruption and spinal cord injury. The management of survivors is difficult and controversial and include no treatment, traction, external immobilization, and internal fixation with fusion.

The dislocation in atlanto-occipital dislocation is reduced by traction. After reduction, a cervical orthotic device can be used to reduce motion and therefore, orthotics allow the atlanto-occipital junction to heal properly. Woodring et al. [8] reported a case in which successive conservative treatment using orthotics was applied and reported no spinal instability months after therapy. However, in their case, stability was secondary to fibrous ankylosis, which they believe does not occur in adults. However, in some cases, surgical reduction may not be possible because of the firm union in the dislocated atlanto-occipital junction during the chronic phase [9]. But, complete ligament healing is difficult to achieve with conservative care, and the risk of re-displacement always exists. For this reason, treatment with internal fixation and arthrodesis is the treatment of choice. Surgical treatment of atlanto-occipital dislocation attempts to achieve immobility at the motion segment to provide stability by eliminating motion at the atlanto-occipital level. In our case, the patient should have been treated with surgery, but he refused it.

The swallowing process is controlled by coordination of the brain stem, cortical central pathways, and cranial nerves. The cranial motor nuclei (the trigeminal motor nucleus, the facial motor nucleus, the nucleus ambiguus, and the hypoglossal nucleus), and the cranial sensory nuclei (the trigeminal sensory nuclei, and the nucleus tractus solitarius) located in the brain stem are involved. Normal swallowing requires intact function of the trigeminal (V), facial (VII), glossopharyngeal (IX), vagus (X), and hypoglossal (XII) cranial nerves. The afferent cranial nerves (V, VII, IX, and X) are stimulated through sensations of pressure, taste, and temperature. The efferent cranial nerves control mastication (V), muscles of the lip and face (VII), muscles of the palate, pharynx, and larynx (IX, X) and muscles of the tongue (XII).

On VFSS, the movements of the soft palate, pharynx, and larynx were decreased in our patient, as was the gag reflex. Therefore, we postulated that there was an injury to the glossopharyngeal and vagus nerves. Additionally, weakness and decreased movement of the tongue indicated an injury to the hypoglossal nerve. However, there was no weakness of mastication, and the facial muscles and facial sensation were intact. We inferred that the trigeminal and facial nerves were intact. Therefore, our patient had injury to the glossopharyngeal, vagus, accessory, and hypoglossal nerves. Accompanying trauma to the lower cranial nerves is possible in atlanto-occipital dislocation. The mechanism of injury to the lower cranial nerves may be axial traction or compression of both the medulla and the nerves as a result of the displacement and instability. In this case, the medullary compression and deficits of the lower cranial nerves (glossopharyngeal, vagus, and hypoglossal nerves) may have caused the swallowing disorders.

We report a rare case of traumatic atlanto-occipital dislocation presenting with dysphagia as the chief complaint. In patients who are diagnosed with atlanto-occipital dislocation, any neurological symptoms should be carefully and rapidly evaluated.

\section{CONFLICT OF INTEREST}

No potential conflict of interest relevant to this article was reported.

\section{ACKNOWLEDGMENTS}

This study was supported by a grant (2010-0005602) from the National Research Foundation of Korea.

\section{REFERENCES}

1. Ehlinger M, Charles YP, Adam P, Bierry G, Dosch JC, Steib JP, et al. Survivor of a traumatic atlanto-occipital dislocation. Orthop Traumatol Surg Res 2011;97:33540.

2. Diagnosis and management of traumatic atlantooccipital dislocation injuries. Neurosurgery 2002; 50(3 
Suppl):S105-13.

3. Traynelis VC, Marano GD, Dunker RO, Kaufman HH. Traumatic atlanto-occipital dislocation: case report. J Neurosurg 1986;65:863-70.

4. Olszewski J. Causes, diagnosis and treatment of neurogenic dysphagia as an interdisciplinary clinical problem. Otolaryngol Pol 2006;60:491-500.

5. Harris JH Jr, Carson GC, Wagner LK. Radiologic diagnosis of traumatic occipitovertebral dissociation: 1. Normal occipitovertebral relationships on lateral radiographs of supine subjects. AJR Am J Roentgenol 1994;162:881-6.

6. Fujimura Y, Nishi Y, Chiba K, Kobayashi K. Prognosis of neurological deficits associated with upper cervical spine injuries. Paraplegia 1995;33:195-202.

7. Cognetti DM, Enochs WS, Willcox TO. Retropharyngeal pseudomeningocele presenting as dysphagia after atlantooccipital dislocation. Laryngoscope 2006;116:1697-9.

8. Woodring JH, Selke AC Jr, Duff DE. Traumatic atlantooccipital dislocation with survival. AJR Am J Roentgenol 1981;137:21-4.

9. Takayasu M, Hara M, Suzuki Y, Yoshida J. Treatment of traumatic atlanto-occipital dislocation in chronic phase. Neurosurg Rev 1999;22:135-7. 